\title{
圆雉破碎机破碎质量的粒度和粒形数学建模*
}

\author{
马彦军 ${ }^{1}$ 范秀敏 ${ }^{1,2}$ 何其昌 ${ }^{1}$ 董 钢 ${ }^{1}$ \\ (1. 上海交通大学机械工程与动力学院 上海 200240; \\ 2. 上海交通大学机械系统与振动国家重点实验室 上海 200240)
}

\begin{abstract}
摘要: 为定量分析计算圆雉破碎机的破碎物料质量以支持产品的优化设计, 基于层压破碎理论对圆雉破碎机破碎腔进行分层, 建立改进的圆锥破碎机破碎过程的操作模型, 深入研究破碎产品粒度分布模型和粒形的计算方法, 将粒形模型与粒度分布模 型相结合来描述层压破碎过程中物料破碎的本质原因。利用岩石力学试验系统对层压破碎过程进行试验, 建立以压缩比和粒 度分布系数为参数的粒度的选择函数、破碎函数和粒形的选择函数、破碎函数的数学模型。结合 PYGB1121 型圆雉破碎机 实际结构参数及工作参数, 对其破碎过程中的物料粒形和粒度的变化进行求解, 得到圆锥破碎机的粒度分布曲线和针片率随 压缩比和粒度分布系数变化的关系, 为圆雉破碎机破碎过程中破碎生产率的动态优化提供理论模型。
\end{abstract}

关键词：圆锥破碎机 层压破碎 粒形 针片率 粒度分布

中图分类号: TD451

\section{Mathematical Modeling of Particle Size and Particle Shape of Crushing Quality for Cone Crusher}

\author{
MA Yanjun ${ }^{1}$ FAN Xiumin $^{1,2}$ HE Qichang ${ }^{1}$ DONG Gang ${ }^{1}$
}

(1. School of Mechanical Engineering, Shanghai Jiao Tong University, Shanghai 200240;

2. State Key Laboratory of Mechanical System and Vibration, Shanghai Jiao Tong University, Shanghai 200240)

\begin{abstract}
In order to quantitatively analyze and calculate crushing quality and support for cone crusher optimization design, an improved cone crusher manipulation model based on the inter-particle breakage theory is built by studying the crushing process of cone crushers, and calculation methods of particle size distribution and particle shape are under studied. The combination of the particle size distribution model and the flakiness rate model for predicting particle shape, uncovers the essential causes of inter-particle breakage of cone crushers. A rock mechanics testing system is used to do experiment to study the inter-particle breakage process of cone crushers. Mathematical models of particle size selection function, particle size breakage function, particle shape selection function and particle shape breakage function are established and parameterized by compression ratio and particle size distribution. With structural parameters and working parameters of the PYGB1121 cone crusher as input, the variation of the particle shape and size can be calculated, and the particle size distribution curve and the flakiness changed with compression ratio and production size distribution are obtained. The outcome provides theoretical models for dynamic optimization of crushing process.
\end{abstract}

Key words: Cone crusher Interparticle breakage Particle shape Flakiness index Size distribution

\section{0 前言}

圆雉破碎机是岩石物料破碎的主要设备之一， 物料在破碎腔中由动雉偏心运动的挤压而破碎成小 颗粒，该设备广泛用于矿山、治炼、水利、公路、

*高等学校博士学科点专项科研基金资助项目 (20090073110038)。 20121205 收到初稿, 20130220 收到修改稿
铁路和化工等部门, 并在相应的生产工艺中起到了 关键作用 ${ }^{[1]}$ 。其中破碎后产品的粒度和粒形是评价 圆雉破碎机性能的主要指标, 破碎产品粒度和粒形 的好坏直接影响到生产厂家的经济效益, 体现了圆 雉破碎机性能的优劣 ${ }^{[2]}$ 。在混凝土骨料与沥青等建 筑行业中, 混凝土骨料中针片状颗粒的增加会导致 建筑整体抗压强度的下降, 并增加建筑过程中的水 泥消耗量; 同样, 在沥青混合料中, 针片状颗粒的 
增加和粒度大小的不均也会导致混合物整体密度与 抗压、抗拉强度的下降 ${ }^{[3]}$; 国内外对破碎机设计理 论的研究, 其目标也是为提高产品中立方状颗粒的 质量分数 ${ }^{[4]}$ 。由此可见, 针片状颗粒的多少和颗粒 的粒度分布对于破碎产品质量的优劣具是一项重要 指标, 而针片率则用于衡量产品含有针片状颗粒的 多少。此外, 圆雉破碎机工作过程中腔形发生磨损 和喂入物料不均等因素会导致破碎机破碎质量降 低, 对圆雉破碎机破碎产品的粒度和粒形的数学表 达模型的研究, 可以做到对粒度、粒形的计算, 为 破碎机破碎过程的结构参数动态调整来优化破碎产 品质量提供依据, 因而具有重要的理论意义和使用 价值。

国外已有学者在粒形和粒度方面开展研究。 EVERTSSON $^{[5-6]}$ 提出了物料在破碎腔内的整个破 碎过程是由一系列挤压破碎事件构成的, 并由此建 立了粒度预测的模型, 通过选择函数和破碎函数去 描述产品粒度。在产品粒形的研究方面, 国内外大 部分学者都是通过现场试验, 分析影响粒形的主要 因素来建立模型, 这些模型必然与破碎机腔型和相 关设置的工作参数有很大的关系。BOUQUETY 等 ${ }^{[3]}$ 提出了破碎机的工作参数和喂入物料的分级对产品 的针片率的影响。BENGTSSON 等 ${ }^{[7-8]}$ 建立了描述破 碎产品针片率的经验模型, 该模型是喂入物料的平 均粒度、产品粒度和闭边排料口尺寸(Closed side setting, CSS)等参数的函数, 但与层压破碎中的重要 参数 “粒度分布系数和压缩比” 没有关联, 这就造 成无法用层压破碎理论的描述过程去求解产品的粒 形。DONG 等 ${ }^{[9-10]}$ 将产品的针片率经验模型与粒度 模型结合起来用于预测物料的针片率，该模型将一 个经验模型用于层压破碎理论模型, 其适用范围有 一定的限制。BENGTSSON 等 ${ }^{[11}$ 提出了利用粒形的 选择函数和破碎函数对破碎机产品的针片率进行预 测, 为圆锥破碎机破碎产品粒度和粒形预测提供了 理论基础。

本文基于 BENGTSSON 等 ${ }^{[11]}$ 提出的理论, 并利 用 RMT-150B 岩石力学系统对圆雉破碎机层压破碎 过程进行模拟试验研究, 提出并建立考虑粒度分布 系数和压缩比的粒度和粒形的选择函数和破碎函数 数学模型, 实现圆锥破碎机破碎产品的粒度和粒形 的预测, 从而为圆雉破碎机破碎过程的动态优化和 圆雉破碎机的研发提供理论模型。

\section{1 圆锥破碎机破碎过程的操作模型}

在圆雉破碎机破碎工作过程中, 动雉部随偏心
轴套的旋转而围绕破碎腔及定锥的中心线做回旋运 动, 如图 1 所示, 可知动雉不断地在开边和闭边两 极限位置间做往复运动, 其运动实质上是以球面轴 承球面中心, 即悬挂点 $O$ 为圆心的简谐运动。

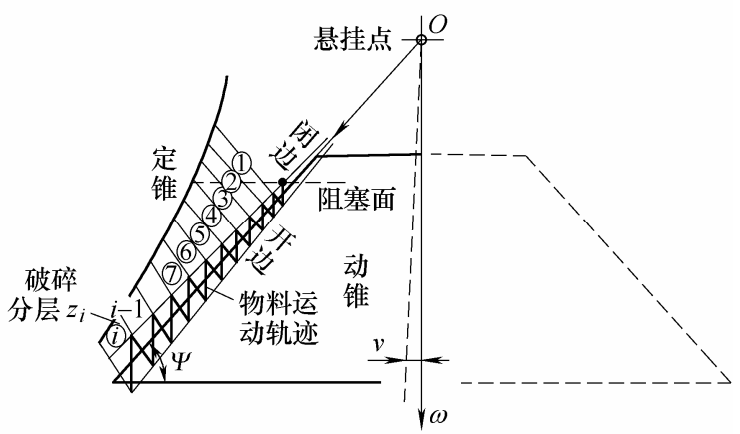

图 1 圆雉破碎机破碎腔分层特性

散体物料从破碎机给料口进入破碎腔, 在挤压 过程中受挤压破碎力不断增大而破碎，破碎壁到达 闭边位置后再次后撤，物料破碎后的若干子颗粒将 再次自由下落, 并重复历经上述过程, 最后经排料 口排出破碎腔, 由破碎腔阻塞面出发, 根据圆雉破 碎机的运动学模型, 可依次求出物料颗粒在通过破 碎腔各破碎层时的详细下落轨迹 ${ }^{[12]}$ 。同时，依据固 定质量原理和层压破碎原理, 散体物料颗粒在破碎 腔内的各破碎层受到、且仅受到一次挤压破碎作用， 并且保证破碎腔各破碎层物料通过质量相等可知, 物料颗粒在破碎腔内的运动轨迹, 即为破碎腔内各 破碎层的分层信息。图 1 即为采用上述方法, 描述 圆锥破碎机的破碎腔内物料下落过程以及破碎腔分 层。圆锥破碎机破碎过程的操作模型揭示了散体物 料破碎的本质过程, 该操作模型是对破碎产品的粒 度和粒形模型建模的重要理论基础, 而破碎过程的 操作模型是基于总体平衡模型和破碎设备矩阵模型 建立的, 总体平衡模型由学者 BROADBENT 及 CALLCOTT 于 1956 首先提出, 破碎过程是由一系 列的破碎事件构成, 每一次的破碎给料均为上一破 碎事件的排料，每次破碎事件均可以用选择函数和 破碎函数来描述, 破碎设备矩阵模型是由 $\mathrm{LYNCH}$ 于 1977 年在矿物破碎与磨碎循环中进行完善的 ${ }^{[13]}$ 。 BENGTSSON 等 ${ }^{[11]}$ 将破碎产品的粒度和粒形通过 粒度选择函数、粒度破碎函数、粒形的选择函数和 粒形的破碎函数所构成的矩阵模型进行描述。基于 总体模型和 BENGTSSON 的研究成果, 结合圆雉破 碎机破碎过程, 建立如图 2 所示的圆雉破碎机破碎 层的操作模型框图。

图 2 中, $Z_{N}$ 为破碎腔的第 $N$ 分层, $\sigma$ 为粒度分 布系数, $(s / b)_{N}$ 为第 $N$ 层的压缩比, $E_{N}$ 为第 $N$ 层破 


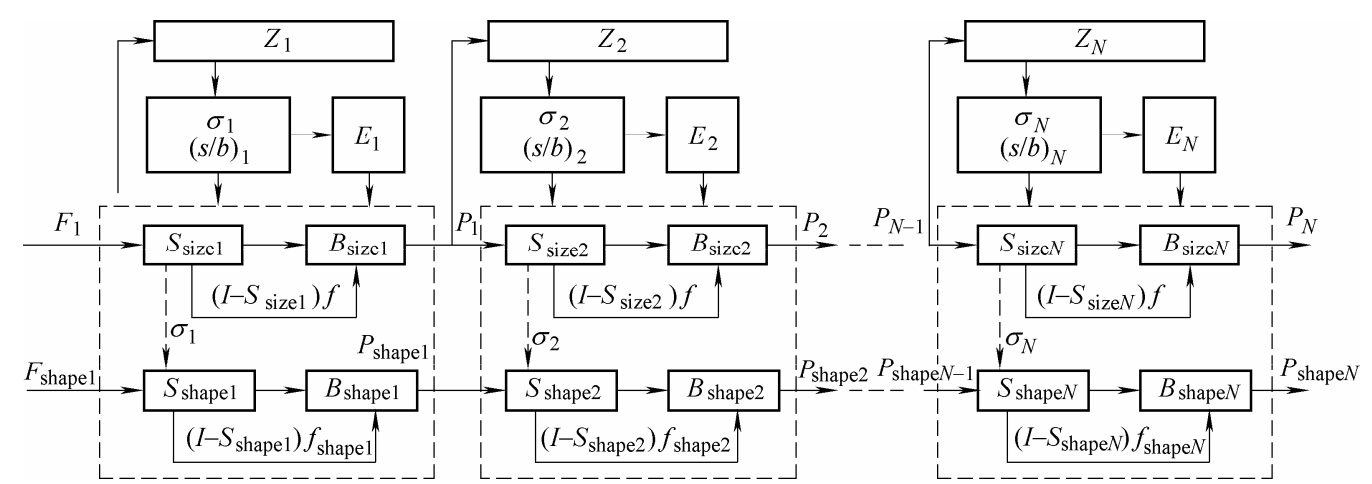

图 2 圆雉破碎机散体物料总体破碎过程

碎过程所需的能耗, $F_{1}$ 为给料中颗粒的粒度分布, $F_{\text {shape } 1}$ 为给料中针片状的粒度分布, $P_{N}$ 为破碎后产 品中的第 $N$ 次所排物料的粒度分布, $P_{\text {shape } N}$ 为第 $N$ 次所排物料中针片状颗粒的粒度分布, $S_{\text {size }}$ 为粒度 的选择函数, $B_{\text {size }}$ 为粒度的破碎函数, $S_{\text {shape }}$ 为粒形 的选择函数, $B_{\text {shape }}$ 为粒形的破碎函数。总体平衡模 型的数学表达形式如式(1)所示 ${ }^{[5]}$

$$
\left(\begin{array}{c}
P_{1} \\
P_{1} \\
P_{1} \\
\vdots \\
P_{N}
\end{array}\right)=\left(\begin{array}{ccccc}
B_{11} & 0 & 0 & \cdots & 0 \\
B_{21} & B_{22} & 0 & \cdots & 0 \\
B_{31} & B_{32} & B_{33} & \cdots & 0 \\
\vdots & \vdots & \vdots & & \vdots \\
B_{N 1} & B_{N 2} & B_{N 3} & \cdots & B_{N N}
\end{array}\right)\left(\begin{array}{c}
F_{1} \\
F_{1} \\
F_{1} \\
\vdots \\
F_{N}
\end{array}\right)
$$

式中, $F_{N}$ 为给料中的第 $N$ 粒级物料所占的质量分 数, $B_{N N}$ 为破碎算子。从式(1)可得粒级 $P_{N}$ 的破碎产 品可从 $F_{1} \sim F_{N}$ 粒级的给料中获得, 表明某粒级破碎 产品只能从与其粒级粗细相同或更粗粒级的给料的 破碎中获得。根据破碎破碎层的操作模型图 2, 可 以得到其数学模型矩阵表达式如式(2)、(3)所示, $P_{N-1}$ 为第 $N$ 层破碎事件给料的粒度分布

$$
\begin{gathered}
P_{N}=\left[B_{\text {size } N} S_{\text {sizeN }}+\left(I-S_{\text {sizeN }}\right)\right] P_{N-1} \\
P_{\text {shapeN }}=\left[B_{\text {shape } N} S_{\text {shape } N}+\left(I-S_{\text {shape } N}\right)\right] P_{\text {shape }(N-1)}
\end{gathered}
$$

根据固定质量原理和层压破碎原理对破碎腔 进行分层研究, 并将粒形模型与粒度分布模型相结 合来描述层压破碎过程, 建立圆锥破碎机过程操作 模型, 该模型是压缩比和粒度分布系数的函数, 并 不受圆雉破碎机的结构参数和工作参数的影响, 可 以基于该模型对圆雉破碎机破碎产品的性能进行深 入研究。

\section{2 散体物料的层压破碎试验}

对于物料的挤压破碎过程而言, 其在破碎过程 中所表现的内在与外在特征, 应当只与物料的初始 状态和挤压过程的特征参数相关。以往的研究成果

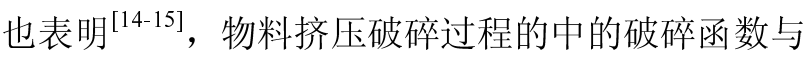
选择函数, 仅与压缩比 $\varepsilon$ 和粒度分布系数 $\sigma$ 相关。压 缩比表示物料受到的压缩程度, 粒度分布系数是 描述散体物料在层压破碎中粒度分布均匀性对选择 函数的影响指数, 可有效地描述各粒级散体物料的 分布情况, 即散体物料群内颗粒尺寸的均匀程度, 其计算过程如式(4) (6)所示

$$
\begin{gathered}
\varepsilon=s / b \\
\sigma=\left[\sum_{j=1}^{m} p_{j}\left(\bar{d}_{j}-\bar{d}\right)^{2}\right]^{1 / 2} / \bar{d} \\
\bar{d}=\sum_{j=1}^{m} p_{j} \bar{d}_{j}
\end{gathered}
$$

式中, $\varepsilon$ 为压缩比, 表示物料受压缩的程度, 压缩 比越大说明物料的体积被压缩的越大。 $b$ 为排料口 尺寸 $\sigma$ 为粒度分布系数, $p_{j}$ 为粒级 $j$ 内散体物料所 占百分比, $\bar{d}_{j}$ 为粒级 $j$ 内散体物料的平均粒度、 $\bar{d}$ 为各粒级平均粒度。

\section{1 试验过程}

为了实现圆锥破碎机产品粒度和粒形的数学 建模, 并对破碎物料质量的数值求解, 必须对破碎 腔各个破碎层选择函数和破碎函数模型进行深入的 探讨。本文采用国产 RMT-150B 岩石力学试验机对 散体物料层压破碎试验进行研究如图 3 所示。

图 3a 3c 所示, 分别为石子压碎仪、压缩比定 义简图和标准石子篎，层压破碎试验前的散体物料 高度为 $b$, 对物料施加压力 $p$, 进给压缩量 $s$, 石子 压碎仪内岩石散体物料发生层压破碎，施加不同的 位移以实现不同压缩比的试验工况，之后利用新型 标准篮对层压破碎的破碎产品进行篎分分析，得到 一系列的破碎产品粒度和针片率的数据, 图 $3 \mathrm{~d}$ 为 RMT-150B 岩石力学试验系统, 利用该系统对散体 物料进行挤压破碎，该过程的应力应变数据可以通 过传感器测定，并可以得到载荷位移关系图和应力 应变关系图。 

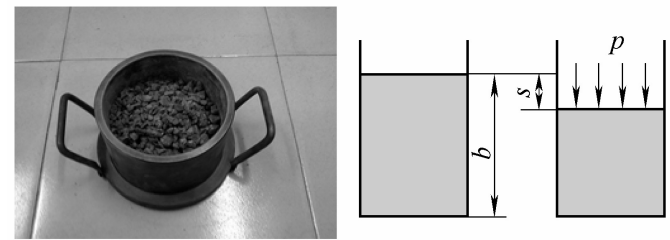

(b) 压缩比的定义

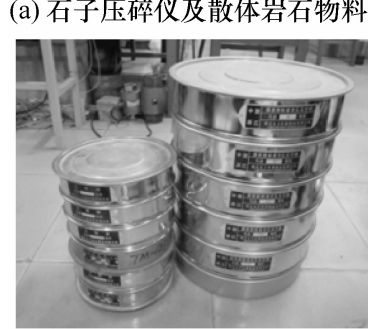

(c) 标准石子篮

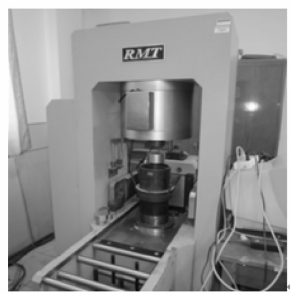

(d) RMT-150B 岩石 力学试验系统

图 3 RMT-150B 岩石力学试验系统及试验辅助设备

基于对层压破碎过程相关影响参数的分析和 研究, 选取物料粒度分布系数和压缩比作为主要试 验参数, 基于破碎装备工作过程中两参量的大致范 围, 选取试验水平值, 并进行试验设计。利用 RMT-150B 岩石力学试验系统对石子压碎仪中的散 体物料进行层压破碎试验, 模拟圆锥破碎机破碎腔 中各破碎层散体物料的层压破碎过程，从而得到一 系列的试验数据, 通过统计回归分析得到破碎腔各 破碎层选择函数和破碎函数模型。如图 4 所示, 将 散体物料放入石子压碎仪中，并对压柱施加压力 $F_{N}$, 使得每次破碎试验中的进给量为 $s_{1}, s_{2}, \cdots$, $s_{N}$, 并利用标准篎进行篮分, 从而利用一系列的层 压破碎试验模拟圆锥破碎机整个破碎腔对物料的层 压破碎过程。层压破碎试验中所使用的散体物料为 取自四川成都矿业的玄武岩矿石, 散体物料为鄂式 破碎机破碎而得到的，其粒度范围为 $19.0 \sim 26.5$ $\mathrm{mm}$, 各种物料的质量大约为 $150 \mathrm{~kg}$, 经测量玄武 岩密度为 $2920 \mathrm{~kg} / \mathrm{m}^{3}$, 其试验流程如图 5 所示。

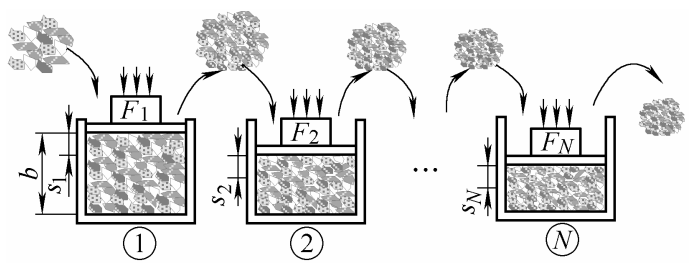

图 4 散体物料层压破碎过程试验原理

散体物料层压破碎试验的具体步骤如下所述。

(1) 取 $19.0 \sim 26.5 \mathrm{~mm}$ 粒径范围的物料, 将物料混 合均匀后装入石子压碎仪中, 对压碎仪进行振动, 在夯 实的过程中保持物料表面平整，高度差不超过 $1 \mathrm{~mm}$ 。

(2) 将装有物料的石子压碎仪放在 RMT-150B 试验力学系统上, 根据压缩比和夯实后物料的高度, 确定试验的压缩进给量, 并设置合适的进给量和极

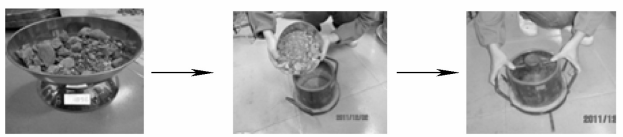

(a) 配比填入物料

(b) 填入物料

(c) 夯实物料

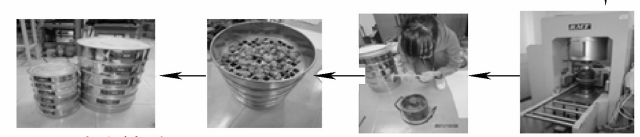

(g) 不同级别的篮分

(f) 篮分物料 (e) 测量压缩

(d) 压缩物料

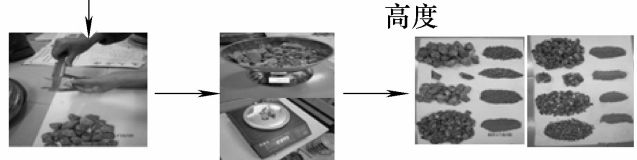

(h) 测量粒形 (i) 称量各粒级的质量 (j) 篮分后不同粒度物料

图 5 散体物料层压破碎试验流程

限压力值，保证试验的顺利进行。

(3) 试验结束后, 测量压碎后物料在石子压碎 仪中的高度, 并对挤压后的物料进行篮分, 并用电子 称对不同粒级物料进行称重, 得到层压破碎篮下粒 度分布数据和相应的曲线。

（4）利用游标卡尺法对 $4.75 \mathrm{~mm}$ 以上的粒级进 行针片率的测量, 得到不同粒径的针片状颗粒的质 量分数。

（5）把篎分后的散体物料混合均匀，重装入石 子压碎仪中，夯实散体物料并使其高度为上次挤压 破碎末时的高度。

(6) 以相同进给压缩比对步骤(5)中的散体物料 进行层压破碎试验, 记录相应的试验数据并对破碎 产品进行篮分分析。

(7) 重复试验步骤(1) (6)若干次, 直到由于 RMT-150B 岩石力学试验系统最大载荷限制导致无 法再对散体物料进行层压破碎为止。

（8）改变进给压缩比分别为 $0.05 、 0.08 、 0.10$ 、 0.14 和 0.18 , 重复步骤(1) (7), 并记录试验数据并 对破碎成品进行篎分, 得到试验结果如表 1 所示。

\section{2 试验数据分析}

\subsection{1 粒度的选择函数和破碎函数}

粒度的选择函数模型是关于破碎腔各破碎层 进给压缩量和相应散体物料粒度分布系数的函数, 当散体物料层压破碎过程中粉末状破碎产品所占比 例较大，在破碎工作粉末中状对粗颗粒散体物料形 成保护时，必须考虑粒度分布系数对选择函数的影 响。对上述试验数据进行统计回归分析，考虑粒度 分布系数对层压破碎选择过程的影响, 可以建立选 择函数模型和破碎函数模型, 基于查默斯理工大学 EVERTSSON 等 ${ }^{[5,14,16]}$ 提出的选择函数和破碎函数 模型, 对试验数据进行拟合, 得到如式(7)、(8)所示 的选择函数模型 
表 1 层压破碎试验结果

\begin{tabular}{|c|c|c|c|c|c|c|c|c|c|c|}
\hline 序号 & $\begin{array}{c}\text { 散体物料 } \\
\text { 粒度范围/mm }\end{array}$ & $\begin{array}{c}\text { 破碎前物料 } \\
\text { 高度 } b / \mathrm{mm}\end{array}$ & $\begin{array}{c}\text { 进给压缩量 } \\
s / \mathrm{mm}\end{array}$ & $\begin{array}{c}\text { 实际压缩量 } \\
s_{1} / \mathrm{mm} \\
\end{array}$ & $\begin{array}{c}\text { 压缩比 } \\
s / b\end{array}$ & $\begin{array}{l}\text { 选择 } \\
\text { 函数 } S\end{array}$ & $\begin{array}{c}\text { 计算选择 } \\
\text { 函数 } S^{\prime}\end{array}$ & $\begin{array}{c}\text { 粒度分布 } \\
\text { 系数 } \sigma\end{array}$ & $\begin{array}{c}\text { 影响 } \\
\text { 因子 } T\end{array}$ & $\begin{array}{l}\text { 针片 } \\
\text { 率 } Z \\
\end{array}$ \\
\hline $1-1$ & $19.0 \sim 26.5$ & 71 & 5.68 & 5.68 & 0.080 & 0.231 & 0.252 & 0.218 & 1.00 & 0.067 \\
\hline $1-2$ & $0 \sim 26.5$ & 65.32 & 5.22 & 5.21 & 0.150 & 0.420 & 0.477 & 0.336 & 0.88 & 0.032 \\
\hline $1-3$ & $0 \sim 26.5$ & 60.1 & 4.80 & 4.80 & 0.220 & 0.427 & 0.649 & 0.490 & 0.66 & 0.031 \\
\hline $1-4$ & $0 \sim 26.5$ & 55.3 & 4.42 & 2.32 & 0.250 & 0.191 & 0.720 & 0.559 & 0.27 & 0.037 \\
\hline $2-1$ & $19.0 \sim 26.5$ & 71.0 & 9.90 & 9.90 & 0.140 & 0.503 & 0.439 & 0.375 & 1.00 & 0.055 \\
\hline $2-2$ & $0 \sim 26.5$ & 61.1 & 8.55 & 7.47 & 0.245 & 0.304 & 0.700 & 0.521 & 0.44 & 0.030 \\
\hline $3-1$ & $19.0 \sim 26.5$ & 71.0 & 12.80 & 12.80 & 0.180 & 0.516 & 0.548 & 0.420 & 1.00 & 0.037 \\
\hline $3-2$ & $0 \sim 26.5$ & 58.2 & 10.48 & 5.41 & 0.260 & 0.304 & 0.725 & 0.524 & 0.42 & 0.026 \\
\hline $4-1$ & $19.0 \sim 26.5$ & 71.0 & 3.55 & 3.55 & 0.050 & 0.074 & 0.148 & 0.129 & 1.00 & 0.079 \\
\hline $4-2$ & $0 \sim 26.5$ & 67.45 & 3.37 & 3.37 & 0.097 & 0.199 & 0.309 & 0.209 & 0.64 & 0.062 \\
\hline $4-3$ & $0 \sim 26.5$ & 64.08 & 3.20 & 3.20 & 0.140 & 0.369 & 0.446 & 0.320 & 0.83 & 0.063 \\
\hline $4-4$ & $0 \sim 26.5$ & 60.88 & 3.04 & 3.03 & 0.185 & 0.383 & 0.562 & 0.412 & 0.68 & 0.050 \\
\hline $4-5$ & $0 \sim 26.5$ & 57.84 & 2.89 & 2.88 & 0.226 & 0.267 & 0.660 & 0.513 & 0.40 & 0.034 \\
\hline $4-6$ & $0 \sim 26.5$ & 54.95 & 2.75 & 1.88 & 0.253 & 0.173 & 0.717 & 0.569 & 0.24 & 0.027 \\
\hline $5-1$ & $19.0 \sim 26.5$ & 70.0 & 7.00 & 7.00 & 0.100 & 0.342 & 0.317 & 0.276 & 1.00 & 0.061 \\
\hline $5-2$ & $0 \sim 26.5$ & 63.0 & 6.30 & 6.30 & 0.190 & 0.438 & 0.574 & 0.430 & 0.76 & 0.033 \\
\hline $5-3$ & $0 \sim 26.5$ & 56.7 & 5.67 & 4.22 & 0.250 & 0.255 & 0.712 & 0.536 & 0.36 & 0.027 \\
\hline
\end{tabular}

$$
T=-142 \sigma^{2}+1.116 \sigma+0.9686
$$

$S(s / b, \sigma)=S(s / b) T(\sigma)=15.3917(s / b)^{2} \sigma^{2}-$

$4.1471(s / b)^{2} \sigma-3.5993(s / b)^{2}-16.2863(s / b) \sigma^{2}+$ $4.3881(s / b) \sigma+3.8085(s / b)+0.1613 \sigma^{2}-$

$$
0.0435 \sigma-0.0377
$$

式中 $T$ 一一影响因子

$S$ 一一挤压破碎过程的粒度选择函数

挤压破碎过程的破碎函数就是粒度分布函数, 根据试验数据可以得到该模型的系数, 相应破碎函 数模型如式(9)、(10)所示

$$
\begin{gathered}
B\left(x_{N},(s / b)_{N}\right)=\left\{1-\left[\alpha_{3}+\alpha_{4}(s / b)_{N}\right]\right\} x_{N}^{\alpha_{1}+\alpha_{2}(s / b)_{N}}+ \\
{\left[\alpha_{3}+\alpha_{4}(s / b)_{N}\right] x_{N}} \\
\alpha_{1}=33.62 \quad \alpha_{2}=4.635 \quad \alpha_{3}=0.09545 \quad \alpha_{4}=0.4137
\end{gathered}
$$

$$
\begin{gathered}
x_{N}=\frac{\log _{2}\left(X_{N} / x_{\min }\right)}{\log _{2}\left(x_{0} / x_{\min }\right)} \\
x_{0}=x_{\max }=19 \quad x_{\min }=0.075
\end{gathered}
$$

式中 $B$ 一挤压破碎过程的破碎函数 $\alpha_{1}, \alpha_{2}, \alpha_{3}, \alpha_{4}$ 一一拟合系数

$x_{0}$ —标准篮篮分物料确定的最大篮孔值

$x_{\min }$ —散体物料的最小粒径

$x_{\text {max }}$ 一一给料的最大粒径值

$x_{N}$ 一层压破碎物料第 $N$ 层当量粒径

$X_{N}$ 一层压破碎物料第 $N$ 层的粒径
粒度分布系数可有效地描述各粒级散体物料 的分布情况, 当个粒径分布不均匀时, 粒度分布系 数值变大，相应选择函数减小，因此粒度分布系数 可以有效地描述粒度分布不均匀所带来对选择函数 的影响。基于式(7) (10)所示的选择函数模型和破 碎函数模型, 利用 Matlab 对其进行数值计算, 可得 到如图 6、7 所示的选择函数和破碎函数分布图。

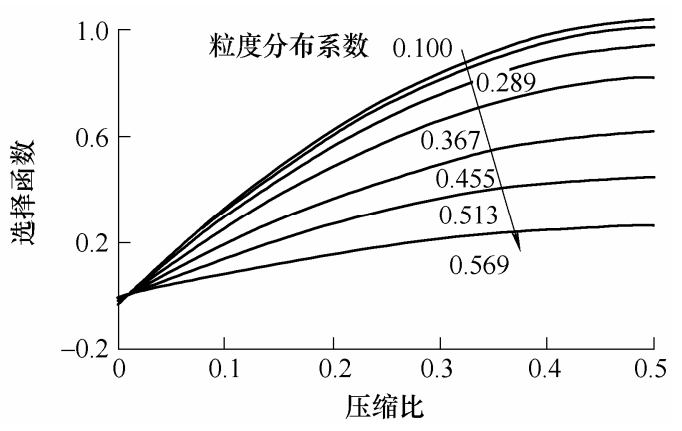

图 6 粒度选择函数模型

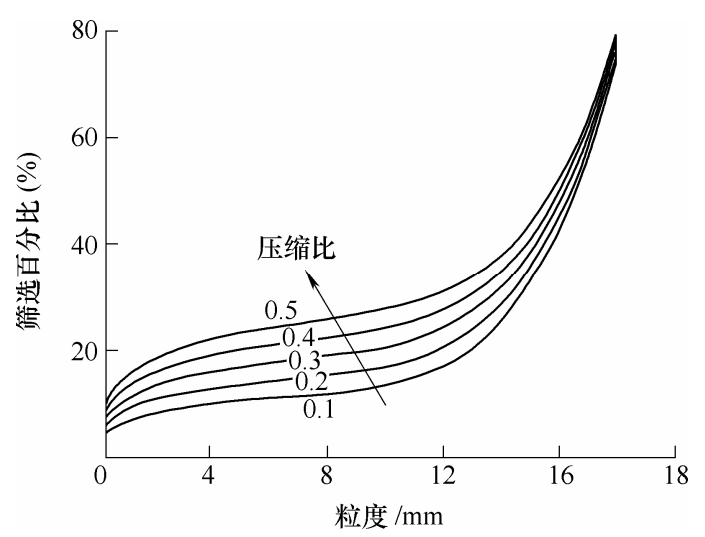

图 7 粒度破碎函数模型 
从图 6 可以得到, 随着粒度分布系数的增加, 其选择函数的曲率降低, 这是由于粒度分布系数增 加时, 也即粒度大小分布更加不均, 使得层压破碎 过程中细粒对粗粒起破碎保护作用而导致部分物料 得不到破碎, 导致选择函数的曲率降低。图 7 则表 明随着压缩比的增加, 某粒度大小的篎下百分比也 是增加的, 说明压缩比的增加有利于降低产品的粒 度大小。

\subsection{2 粒形的选择函数和破碎函数}

根据国标 T3011-2005 粗集料针片状颗粒质量 分数试验标准, 做出两个假设: 针片状颗粒质量分 数测量方法采用的是游标卡尺法, 用于测量 4.75 $\mathrm{mm}$ 以上的粗物料颗粒的针状及片状质量分数; 粒 径接近 0 时, 其针片率质量分数为零, 即 $x_{N}=0$ 时, $y=0$ 。

对上述试验数据进行统计回归分析, 基于 BENGTSSON 相关学者 ${ }^{[11]}$ 提出粒形的选择函数和 破碎函数模型, 对试验数据进行拟合, 得到如式(11) 所示不考虑粒度分布的粒形选择函数模型

$$
S(s / b)=-0.1886(s / b)^{-0.6}+1.316
$$

在试验设计中, 由于料层比较厚, 夯实的过程 对其有一定的影响, 所以对压缩比小于 0.05 的数据 不予考虑。根据以上假设对粒形破碎函数的形式进 行了修正, 对试验数据统计回归得到该模型的系数, 相应粒形破碎函数模型如式(12)、(13)所示

$$
\begin{gathered}
B\left(x_{N},(s / b)_{N}\right)=\alpha_{3} x_{N}^{\alpha_{1}+\alpha_{2}(s / b)_{N}}+\left[\alpha_{4}(s / b)_{N}{ }^{2}+\right. \\
\left.\alpha_{5}(s / b)_{N}+\alpha_{6}\right] x_{N} \\
\alpha_{1}=240.9 \quad \alpha_{2}=44.92 \quad \alpha_{3}=0.9148 \\
\alpha_{4}=6.34 \quad \alpha_{5}=-3.41 \quad \alpha_{6}=0.5 \\
x_{N}=\frac{\log _{2}\left(X_{N} / x_{\min }\right)}{\log _{2}\left(x_{0} / x_{\min }\right)} \\
x_{0}=x_{\text {max }}=26.5 \quad x_{\text {min }}=4.75
\end{gathered}
$$

基于式(11) (13)所示的选择函数模型和破碎 函数模型, 利用 Matlab 数值仿真工具对其进行数值 计算, 可得到如图 8、9 所示的粒形选择函数和破碎 函数分布图。

从图 8、9 中可以得到, 随着压缩比的增加, 发生破碎的物料越来越多, 压缩比的增加使得粒形 破碎函数的曲率降低。通过试验得到层压破碎过程 的粒度选择函数、粒度破碎函数、粒形选择函数和 粒形破碎函数模型后, 可利用式(2)、(3)所示的层压 破碎过程操作模型对破碎产品粒度和粒形分布进行
仿真研究, 从而实现对破碎产品质量的有效控制, 为圆锥破碎机破碎过程的动态优化奠定理论基础。

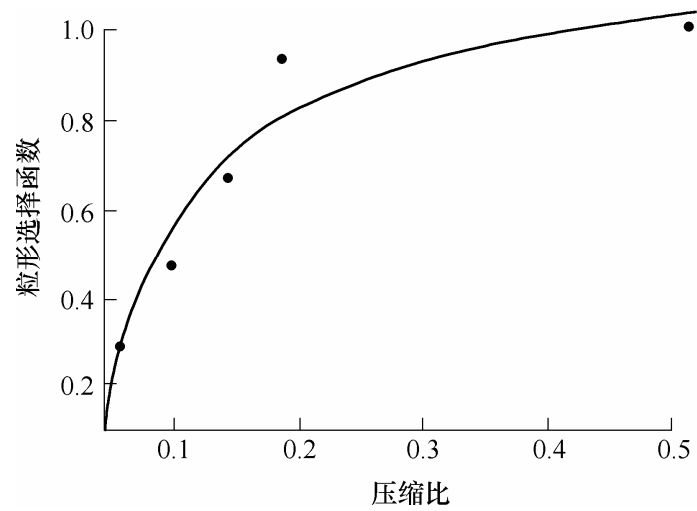

图 8 粒形选择函数模型

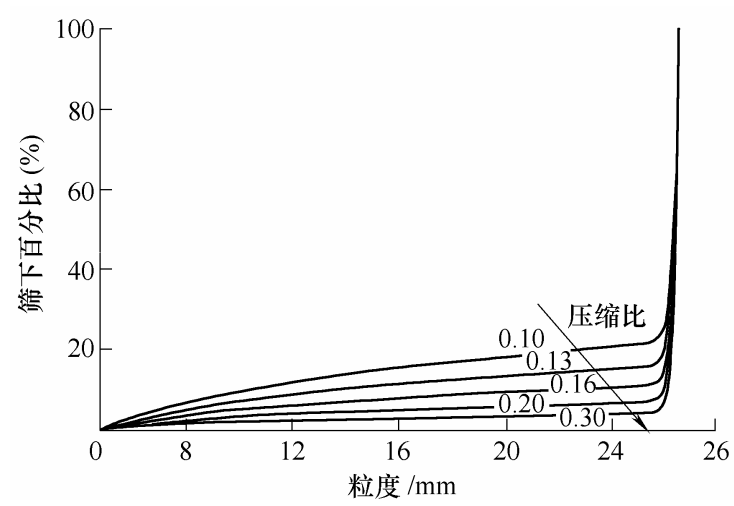

图 9 粒形破碎函数模型

3 基于粒度和粒形模型的圆雉破碎机 破碎性能

以国产的 PYGB1121 型圆雉破碎机为例, 其结 构参数如表 2 所示。

表 2 PYGB1121 的工作及结构参数

\begin{tabular}{cccccc}
\hline 参数 & $\begin{array}{c}\text { 生产率 } \\
Q /(\mathrm{t} / \mathrm{h})\end{array}$ & $\begin{array}{c}\text { 标定排料 } \\
\text { 粒度 } P_{b}(\%)\end{array}$ & $\begin{array}{c}\text { 给料口尺 } \\
\text { 寸 } B_{\mathrm{g}} / \mathrm{mm}\end{array}$ & $\begin{array}{c}\text { 排料口尺 } \\
\text { 寸 } b / \mathrm{mm}\end{array}$ & $\begin{array}{c}\text { 冲程 } \\
S_{\mathrm{t}} / \mathrm{mm}\end{array}$ \\
\hline 数值 & $200 \sim 445$ & 80 & 211 & 20 & 75 \\
\hline
\end{tabular}

利用以上模型对其破碎性能进行求解仿真。从 上述模型可知, 散体物料层压破碎过程选择函数模 型和破碎函数模型均为相应进给压缩比的函数，而 各破碎层进给压缩比关于破碎机工作参数和结构参 数的函数, 选择破碎机工作参数与结构参数作为模 型设计变量, 根据表 2 中的数据, 依据破碎产品粒 度分布计算模型(式(2)、(3)、(7) (10)) 可以求得破 碎腔各层压破碎层排出的物料和最终产品的粒度分 布曲线，如图 10 所示。 


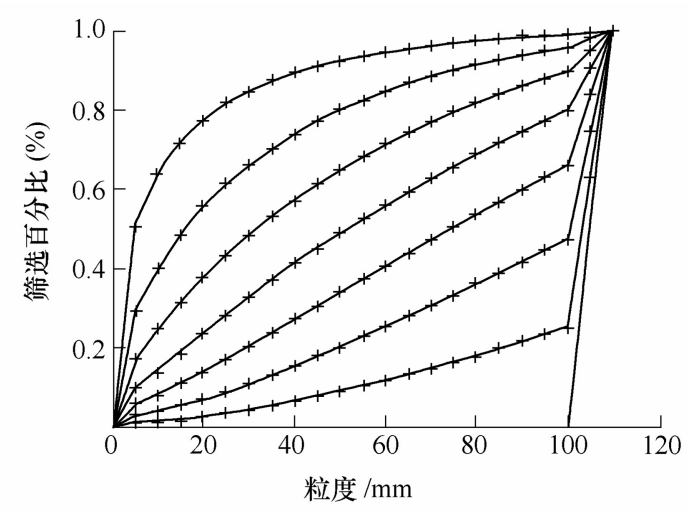

图 10 各破碎层产品粒度分布图

从粒度分布图中可以得到 PYGB1121 的圆雉破 碎机的标定排列粒度接近 $80 \%$, 这个结果与该型号 破碎机的性能数据基本吻合的。利用 Matlab 程序可 以分析针片率与压缩比之间的关系如图 11 所示。

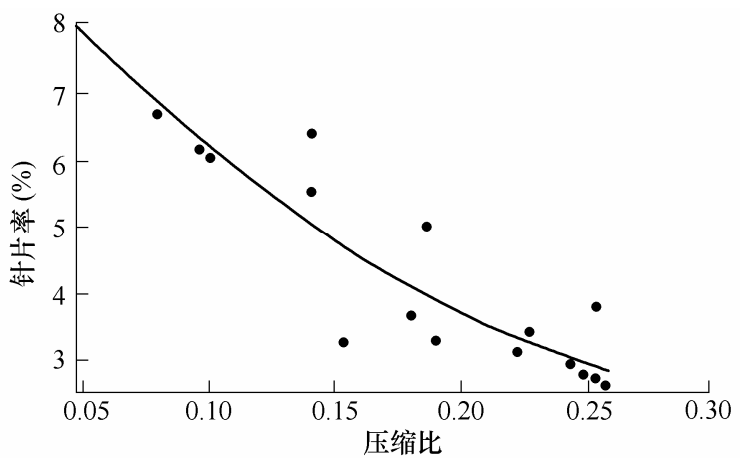

图 11 压缩比对针片率的影响

从图 11 中可以看出随着压缩比的增加针片率 逐渐减少，这也与挤压破碎试验所得到的试验数据 的趋势相吻合。为了得到粒度分布与压缩比对针片 率的影响程度, 对空间变量数据进行分析如图 12 所示。

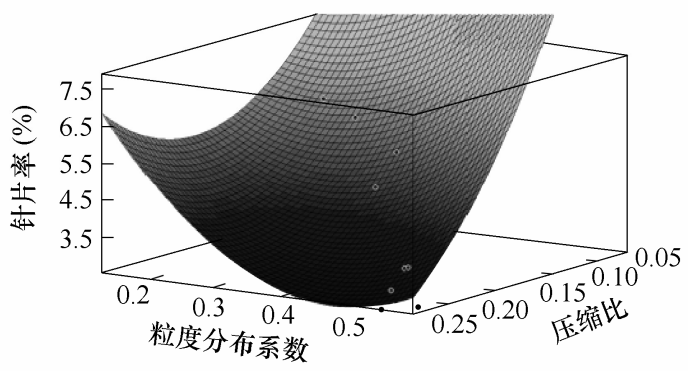

图 12 压缩比和粒度分布对针片率的影响

(粒度为 $4.75 \sim 26.50 \mathrm{~mm}$ )

从图 12 中可以得到, 在破碎过程中较大的压 缩比和粒度分布系数有助于降低针片率的质量分 数, 并且粒度分布系数对针片率的影响相对较小, 而压缩比对其影响较大, 这个与试验数据也是吻合
的。在物料进入破碎机前, 经过篎选或粒度分离工 序后其粒度变得较为均匀, 进入破碎腔后, 物料受 到挤压破碎作用, 开始出现针片状颗粒, 当挤压破碎 事件进一步增加时，包括针片状颗粒在内的部分物 料颗粒开始受到两次或多次挤压作用，针片率的大 小减小并趋于稳定, 可见，在圆雉破碎机工作过程 中，挤压破碎事件发生的频率增加，有利于减少破 碎产品中的针片状颗粒。从图 12 中也可以观察到, 在压缩比为 0.1 时, 粒度分布系数为 0.56 时的产品 的针片率为 $4.46 \%$, 增加压缩比到 0.25 , 粒度分布 系数不变, 则其产品针片率下降到 $4.07 \%$, 在粒度 分布不变时, 针片率大致随压缩比增加呈减少趋势; 该模型针片率的变化趋势与物料层压破碎过程中针 片率的变化趋势基本相符。破碎产品的实际粒度分 布和针片率是稍有偏差的，这些与破碎过程中圆锥 破碎机腔型的磨损、喂入物料粒度粒形的不均和理 论模型的前提假设等因素相关。但总体而言，圆雉 破碎机破碎产品粒度分布和粒形的模型为破碎机破 碎过程的动态优化提供了理论模型, 对研发圆锥破 碎机的动态优化系统具有一定的指导意义。

\section{4 结论}

（1）基于总体平衡模型、破碎矩阵模型, 改进 了圆锥破碎机散体物料总体破碎过程操作模型, 将 破碎产品的粒度和粒形求解过程结合到一起，利用 圆锥破碎机工作参数和结构参数, 分析层压破碎过 程中产品的粒度和粒形, 为有效地求解圆锥破碎机 的破碎性能提供一种理论方法。

(2) 利用挤压破碎试验对破碎机层压破碎过程 进行模拟试验，推导并建立了粒度的选择函数、破 碎函数模型、粒形的选择函数和破碎函数模型及模 型中的各项参数取值, 提出并建立了粒形和粒度与 压缩比之间的函数关系，为圆雉破碎机产品的粒度 和粒形求解提供了计算模型。

(3) 结合 PYGB1121 型圆雉破碎机, 对其破碎 过程中的物料粒形和粒度的变化进行分析, 得到了 圆锥破碎机破碎产品的粒度分布图和针片率随压缩 比、粒度分布系数变化的关系, 结果表明了破碎分 层和压缩比的增加有利用改善产品的粒形和粒度, 为圆雉破碎机破碎过程的工作参数动态调整来优化 破碎产品质量提供理论模型。

\section{参 考 文 献}

[1] 郎宝贤, 郎世平. 破碎机[M]. 北京：治金工业出版社, 2008. 
LANG Baoxian, LANG Shiping. Crusher[M]. Beijing: Metallurgical Industry Press, 2008.

[2] 黄冬明. 挤压类破碎机工作机理和工作性能优化研究 [D]. 上海: 上海交通大学, 2008 .

HUANG Dongming. Research on working mechanism and working performance optimization of compressive crusher[D]. Shanghai: Shanghai Jiao Tong University, 2008.

[3] BOUQUETY M N, DESCANTES Y. Experimental study of crushed aggregate shape $[\mathrm{J}]$. Construction and Building Materials, 2007, 21(4): 865-872.

[4] 黄冬明, 武殿梁, 范秀敏, 等. 圆锥破碎机的多目标规 划设计 [J]. 机械工程学报，2007，43(3)：204-211.

HUANG Dongming, WU Dianliang, FAN Xiumin, et al. Design for multiobjective planning of cone crusher[J]. Chinese Journal of Mechanical Engineering, 2007, 43(3): 204-211.

[5] EVERTSSON C M. Size reduction in cone crushers[C]//Minerals Engineering Conferenle, Falmouth, England, Sep. 22-24, 1999: E1-E30.

[6] EVERTSSON C M. Output prediction of cone crushers[J]. Minerals Engineering,1998, 11(3): 215-231.

[7] BENGTSSON M, SVEDENSTEN P, EVERTSSON C M. Improving yield and shape in a crushing plant[J]. Minerals Engineering, 2009, 22(7-8): 618-624.

[8] BENGTSSON M, EVERTSSON C M. An empirical model for predicting flakiness in cone crushing[J]. International Journal of Mineral Processing, 2006, 79(1): 49-60.

[9] DONG Gang, HUANG Dongming, FAN Xiumin. Cone crusher chamber optimization using multiple constraints[J]. International Journal of Mineral Processing, 2009, 93(2): 204-208.

[10] 董钢, 范秀敏, 黄冬明. 基于产品质量控制预测机制的 圆锥破碎机优化方法 [J]. 机械工程学报, 2010，46(3): 152-157.

DONG Gang, FAN Xiumin, HUANG Dongming. Cone crusher optimization based on quality control and prediction[J]. Journal of Mechanical Engineering, 2010,
46(3): 152-157.

[11] BENGTSSON M, LEE E, EVERTSSON C M. Influence of throw and compression ratio on particle shape-a full scale investigation and laboratory tests[J]. Minerals Engineering, 2010, 23(7): 549-557.

[12] 黄冬明, 范秀敏, 武殿梁, 等. 挤压类破碎机破碎产品 粒度分析[J]. 机械工程学报，2008，45(5)：201-207.

HUANG Dongmin, FAN Xiumin, WU Dianliang, et al. Crushing product size-reduction analysis of compressing crusher[J]. Chinese Journal of Mechanical Engineering, 2008, 45(5): 201-207.

[13] EVERTSSON C M. Modelling of flow in cone crushers[J]. Minerals Engineering, 1999, 12(12) : 1479-1499.

[14] EVERTSSON C M. Cone crusher performance[D]. Sweden: Chalmers University of Techology, 2000.

[15] 董钢, 范秀敏, 张曦, 等. 基于层压破碎理论的圆雉破 碎机破碎力分析及运动学研究[J]. 机械工程学报, 2010, 46(17): 159-164

DONG Gang, FAN Xiumin, ZHANG Xi, et al. Crushing force and kinematics analysis of cone crusher based on interparticle breakage[J]. Journal of Mechanical Engineering, 2010, 46(17): 159-164.

[16] LEE E, EVERTSSON C M. A comparative study between cone crushers and theoretically optimal crushing sequences[J]. Minerals Engineering, 2011, 24(3-4) : 188-194.

作者简介: 马彦军(通信作者), 男, 1982 年出生, 博士研究生。主要研 究方向为机械系统最优化设计、虚拟样机技术。

E-mail:kfmyj@163.com

范秀敏, 女, 1971 年出生, 博士, 教授, 博士研究生导师。主要研究方 向为数字化设计与制造、虚拟现实技术和虚拟样机技术。

E-mail: xmfan@sjtu.edu.cn

何其昌, 男, 1978 年出生, 博士, 讲师。主要研究方向为数字化设计与 制造、虚拟现实技术.

E-mail: heqc@sjtu.edu.cn

董钢, 男, 1981 年出生, 博士研究生。主要研究方向为机械系统最优化 设计、虚拟样机技术。

E-mail: jon.dong@sohu.com 